\section{De víctimas, perpetradores y espectadores: una meta-etnografía de los roles en el ciberbullying}

\author{
Victims, perpetrators, and bystanders: a \\ meta-ethnography of roles in cyberbullying
}

Sobre vítimas, agressores e espectadores: uma
meta-etnografia dos papéis no cyberbullying
Constanza Moretti 1
Damián Herkovits 1

doi: 10.1590/0102-311X00097120

\section{Resumen}

El ciberbullying es una agresión virtual entre pares, cuya prevalencia varía entre el 10\% y el 40\%, según estudios realizados en diferentes países. Gran parte de la bibliografía académica sobre esta agresión tiende a individualizar y medicalizar las causas de la violencia, sin comprender el contexto en el que se desarrolla y los sentidos que adquieren para quienes la ejercen. El objetivo de este trabajo es conocer cuáles son las creencias, valores y prácticas que los adolescentes movilizan en el desempeño de los roles involucrados en las prácticas de ciberbullying. Para llevarlo a cabo se realizó una meta-etnografía, cuyo propósito es producir una sintesis de investigaciones cualitativas, a partir de la interpretación teórica de sus hallazgos fundamentales. El corpus analizado se conformó con 33 artículos seleccionados en las bases de bibliografía científica BVS, PubMed, SciELO y Scopus. En los resultados se describen las expresiones de ciberbullying, los motivos desencadenantes, y las experiencias de adolescentes como personas agresoras, agredidas y observadoras. Tomando como referencia teórica el interaccionismo simbólico, consideramos que el ciberbullying es una expresión singular de la sociabilidad virtual. Se sostiene que es una práctica vinculada con procesos de construcción de identidad, a partir de mecanismos de identificación y oposición con pares, por los cuales también se reproducen y disputan posiciones de reconocimiento en su sociabilidad. En ese proceso, a través del cyberbullying ise sancionan comportamientos que transgreden un orden simbólico dominante sobre el deber ser y estar adolescente.

Ciberacoso; Relaciones Interpersonales; Violencia; Adolescente; Revisión

\author{
Correspondencia \\ C. Moretti \\ Instituto de Salud Colectiva, Universidad Nacional de Lanús. \\ 29 de Septiembre 3901, Remedios de Escalada, Lanús, \\ Buenos Aires 1826, Argentina. \\ mariaconstanzamoretti@gmail.com \\ 1 Instituto de Salud Colectiva, Universidad Nacional de Lanús, \\ Lanús, Argentina.
}




\section{Introducción}

El ciberbullying entre adolescentes es una práctica que ha sido introducida como un problema novedoso en los campos de salud y educación. La bibliografía lo suele definir como una agresión intencional (ya sea verbal, emocional o social), por parte de una persona o grupo, a través de dispositivos electrónicos y de forma repetitiva a lo largo del tiempo, a otra persona que no puede defenderse fácilmente 1,2,3. Esta definición es una adaptación a espacios virtuales del concepto de bullying escolar, tomada a principios del siglo XXI cuando comienza a investigarse el ciberbullying en Estados Unidos y Europa. Sin embargo, diferentes investigadores consideran que esta definición es inacabada o inconsistente con las características del espacio virtual, y que es necesario centrarse en cómo las tecnologías de información y comunicación (TICs) modifican los modos de conceptualizar las agresiones entre adolescentes. Por ejemplo, se considera que la virtualidad construye nuevas formas de desigualdad de poder, sobre todo, por la trascendencia del tiempo y espacio que permite un acceso irrestricto a la víctima, y por la posibilidad de actuar desde el anonimato que habilita agredir sin comprometer la identidad 1,2,3,4,5,6. Otra discusión se dirige a cómo precisar el criterio de repetición, porque no debe pensarse solo como la cantidad de agresiones hacia una misma persona, ya que la reproducción ilimitada de un acto único amplifica el daño cualitativamente 1,2,3,4,5. Estas características, junto a la inscripción de la agresión en el espacio de forma permanente y la participación de una audiencia ampliada, forjan nuevas dinámicas en el ciberbullying que requieren un análisis y teorización particular. Estudios realizados en diferentes países informan que la prevalencia en adolescentes varía entre el $10 \%$ y el $40 \%$, y quienes lo sufren pueden padecer problemas psicosomáticos, depresión, estrés, bajo rendimiento escolar, dificultades para relacionarse con sus pares, e incluso autolesiones e ideación suicida 4,6,7.

La bibliografía señala que en el ejercicio del ciberbullying existen tres roles fundamentales: perpetradores, víctimas o espectadores, y que las personas pueden asumirlos alternadamente según el contexto 5,8,9. Entre estos roles, los espectadores suelen ocupar un papel central, puesto que sus acciones modifican el curso de los sucesos. Esto es, pueden reducir los efectos de los incidentes al ofrecer apoyo a las personas agredidas, reforzar la agresión por unirse al acoso, reproducir el material en cuestión o actuar pasivamente legitimando la acción 10.

Las investigaciones sobre ciberbullying se dirigen principalmente a analizar las diferencias con el bullying presencial 1,2,3, identificar su prevalencia 11,12, construir perfiles epidemiológicos 11,13,14,15, desarrollar modelos predictivos de comportamiento 16,17,18,19, validar escalas de medición 20,21,22,23, establecer consecuencias en la salud 6,7 y evaluar programas de intervención en escuelas 24,25 . Esos estudios tienden a focalizarse en la identificación de atributos personales de los adolescentes que operan como factores de riesgo para explicar el ciberbullying, sin incluir sus perspectivas para comprender el contexto en el que se desarrolla y los sentidos para quienes la ejercen. Por otro lado, las investigaciones cualitativas describen creencias, valores y prácticas de adolescentes que experimentaron ciberbullying desde uno de los roles o sobre la sociabilidad virtual en general, pero no logran abordar las interacciones entre los roles, ni reconstruir la dinámica de las agresiones 26,27,28,29. En este sentido, aún queda pendiente investigar los procesos relacionales y simbólicos que desencadenan el ciberbullying y la articulación con el contexto. Asimismo, el desarrollo de una interpretación teórica que trascienda las singularidades de los casos todavía constituye un desafío para este campo.

A partir de esta lectura, nuestra atención se dirigió a conocer cuáles son las creencias, valores y prácticas recurrentes que los adolescentes movilizan en su accionar en cada uno de los roles que desempeñan en el ciberbullying, cuáles son las características contextuales transversales que los interpelan o no a actuar, y cómo se inscribe en su sociabilidad cotidiana. Nuestro propósito es realizar una síntesis interpretativa de investigaciones cualitativas realizadas en diferentes conjuntos sociales, que permita identificar patrones transversales de las dinámicas del ciberbullying y de la sociabilidad virtual. Para ello, realizamos una meta-etnografía de artículos científicos. La meta-etnografía se diferencia de otras revisiones, porque sintetiza hallazgos a través de traducciones de núcleos de sentido entre investigaciones, con la intención de realizar una nueva interpretación teórica, basada en las perspectivas y experiencias de las personas estudiadas 30 . En este caso, desde el interaccionismo simbólico analizamos al ciberbullying como una expresión singular de la sociabilidad virtual 31,32,33. Así, sostenemos que es una práctica vinculada con procesos de construcción de identidad, a partir de mecanismos de identificación y oposición con pares, por los cuales también se reproducen y disputan 
posiciones de reconocimiento en su sociabilidad 34,35,36,37. En ese proceso, a través del ciberbullying se sancionan comportamientos que transgreden un orden simbólico dominante sobre el deber ser y estar adolescente 38. Por último, desarrollamos qué implicancias tienen los resultados obtenidos para el diseño de políticas sobre ciberbullying en adolescentes.

\section{Metodología}

La meta-etnografía consiste en un tipo de metasíntesis de investigaciones realizadas solo con metodología cualitativa, cuyo proceso está compuesto por siete etapas que se dan de forma simultánea e interactiva 30,39 . Las primeras dos consisten en la identificación del problema de investigación y la selección de estudios relevantes correspondientes para constituir un corpus a analizar. Para identificar los estudios, se consultaron las bases de bibliografía científica BVS, PubMed, SciELO y Scopus por su reconocimiento internacional y la especialidad temática en salud y ciencias sociales. Para seleccionar los términos de búsqueda se realizó una etapa exploratoria en la que se identificaron y probaron un conjunto de palabras que pudieran recuperar estudios sobre ciberbullying. Estas son: online bullying; cyber victimization, online aggression, cyber harassment, cyber abuse, peer victimization AND Internet, stalking AND Internet, cyber space AND violence, social media AND violence, Internet AND violence, social networking $A N D$ violence, sexting AND violence, Facebook AND violence, Twitter Messaging AND violence, hate speech AND Internet. Tras la lectura y análisis de los resultados obtenidos, y la construcción de un corpus preliminar, se identificó que los términos amplios recuperan principalmente otros fenómenos del espacio virtual y que todas las investigaciones que trabajan ciberbullying lo mencionan de esta manera, pese a que discutan su conceptualización. Si bien el descriptor cyberbullying ingresó en 2019 al tesauro $\mathrm{MeSH}$, los investigadores utilizan esa nominalización desde los inicios del siglo XXI 1. Por ello, para la búsqueda final se decidió utilizar solo cyberbullying como un término libre (Tabla 1).

A partir de las citas obtenidas en las búsquedas, seleccionamos los artículos científicos que cumplieran con los siguientes criterios: ser realizados con metodología cualitativa que relevaran el punto de vista de las personas entrevistadas, su objeto de estudio es el ciberbullying, definido de esta manera por los investigadores, y su población de estudio son adolescentes entre 11 y 18 años. Se excluyeron trabajos que lo mencionan de manera secundaria y que evalúan programas de intervención en escuelas. El proceso de construcción del corpus se puede observar en la Figura 1. La composición final del corpus de análisis fue de 33 investigaciones que puede consultarse en el Cuadro 1. El corpus fue evaluado a partir de la herramienta Critical Appraisal Skills Programme (CASP. https://casp-uk.net/wpcontent/uploads/2018/03/CASP-Qualitative-Checklist-2018_fillable_form.pdf), con el propósito de cuestionar la calidad de investigaciones cualitativas. Su evaluación y discusión concluyó que el material revela calidad, en cuanto a la claridad de los registros, y es suficiente para los objetivos propuestos. Puede verse el detalle del análisis en el Cuadro 2.

Tabla 1

Cantidad de citas recuperadas a partir de las bases de datos seleccionadas. Abril, 2019.

\begin{tabular}{lcccc}
\hline $\begin{array}{l}\text { Base de } \\
\text { datos }\end{array}$ & Término de búsqueda & Campo de búsqueda & Filtro & Cantidad de citas \\
\hline BVS & Cyberbullying & Título, resumen, asunto & Artículo & 3.808 \\
PubMed & Cyberbullying & Título, resumen & Artículo & 530 \\
SciELO & Cyberbullying & Resumen & - & 59 \\
Scopus & Cyberbullying & Título, resumen, & Artículo & 1.430 \\
& & palabras-clave & & 5.827 \\
\hline
\end{tabular}

Fuente: elaboración propia a partir de datos de bibliotecas electrónicas BVS, PubMed, SciELO y Scopus. 
Diagrama del proceso de selección del corpus documental.

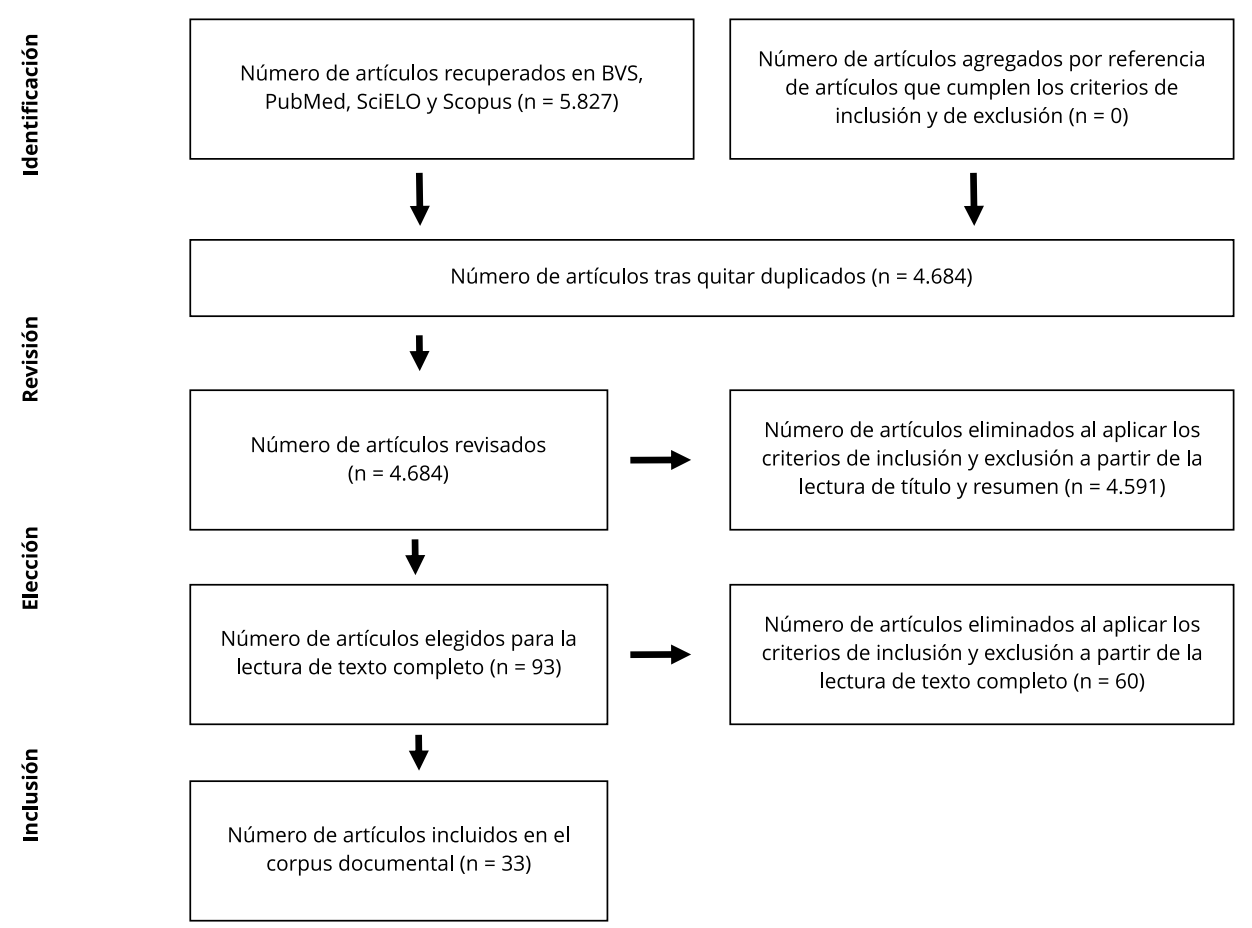

Fuente: elaboración propia a partir de France et al. 39.

En tercer lugar, de los artículos seleccionados se analizaron los apartados de resultados, discusión y conclusiones, con el fin de detectar núcleos temáticos en cada uno de ellos para, en cuarto lugar, comprender cómo se relacionan. En esta lectura, identificamos que en todos los estudios los adolescentes relatan experiencias personales o de sus pares sobre el ciberbullying y la sociabilidad. Las investigaciones suelen focalizarse en un solo rol, y ofrecen resultados que se complementan entre sí. Por ello, dentro de la propuesta meta-etnográfica, realizamos una síntesis a partir de la composición de una "línea argumentativa", que consiste en inferir estructuras de significación de las partes (estudios sobre roles de manera independiente) que permiten comprender un todo (el ciberbullying). Así, se analizan las similitudes y diferencias entre casos y se los integra en un marco interpretativo holístico.

La quinta etapa implica la traducción sistemática de los núcleos identificados anteriormente. La traducción radica en establecer analogías entre los estudios a partir de los significados del texto. No es solo el dato lo que se sintetiza, sino también lo implícito en él. La sexta etapa consistió en la síntesis de las traducciones realizadas entre los estudios, con el objetivo de construir un todo a partir de las partes en función del marco teórico. Por eso, la descripción de los resultados se presenta considerando el corpus como una totalidad, sin hacer mención a los artículos de forma individual. La séptima es la presentación de la síntesis que tiene la intención de ofrecer una nueva interpretación sobre el objeto de estudio que atraviese el conjunto de investigaciones. 


\section{Cuadro 1}

Características del corpus documental.

\begin{tabular}{|c|c|c|c|c|c|}
\hline \multirow[t]{2}{*}{ Autores } & \multirow[t]{2}{*}{ Objetivo } & \multirow[t]{2}{*}{ Metodología } & \multirow[t]{2}{*}{ País } & \multicolumn{2}{|c|}{ Muestra } \\
\hline & & & & Edad (años) & Tamaño \\
\hline Baas et al. 54 & $\begin{array}{l}\text { Explorar el impacto del } \\
\text { ciberbullying, motivos de } \\
\text { agresores y lucha contra } \\
\text { el ciberbullying }\end{array}$ & Entrevistas grupales & Países Bajos & $11-12$ & $\begin{array}{c}\mathrm{N}=28 \text { (13 mujeres, } \\
15 \text { varones })\end{array}$ \\
\hline Berne et al. 27 & $\begin{array}{c}\text { Identificar las } \\
\text { características de } \\
\text { víctimas o agresores en } \\
\text { el ciberbullying dirigido a } \\
\text { la apariencia }\end{array}$ & Entrevistas grupales & Suecia & 15 & $\begin{array}{c}\mathrm{N}=27 \text { (13 mujeres, } \\
14 \text { varones) }\end{array}$ \\
\hline Betts \& Spenser 51 & $\begin{array}{c}\text { Analizar los usos de las } \\
\text { tecnologías y el concepto } \\
\text { de ciberbullying }\end{array}$ & Entrevistas grupales & Reino Unido & $11-15$ & $\begin{array}{c}\mathrm{N}=29 \text { (11 mujeres, } \\
18 \text { varones })\end{array}$ \\
\hline Bowler et al. 52 & $\begin{array}{c}\text { Construir un marco } \\
\text { conceptual generado } \\
\text { por el usuario para } \\
\text { la comprensión del } \\
\text { ciberbullying }\end{array}$ & Entrevistas grupales & EE.UU. & $14-20$ & $\begin{array}{c}\mathrm{N}=9 \text { (6 mujeres, } \\
3 \text { varones) }\end{array}$ \\
\hline Brandau \& Evanson 26 & $\begin{array}{c}\text { Explorar los aspectos } \\
\text { sociales y psicológicos } \\
\text { del ciberbullying desde } \\
\text { el punto de vista de } \\
\text { víctimas }\end{array}$ & $\begin{array}{l}\text { Entrevistas } \\
\text { individuales }\end{array}$ & EE.UU. & $14-21$ & $\begin{array}{c}\mathrm{N}=15 \text { (12 mujeres, } \\
3 \text { varones) }\end{array}$ \\
\hline Bryce \& Fraser 40 & $\begin{array}{c}\text { Analizar las percepciones } \\
\text { y experiencias de } \\
\text { ciberbullying }\end{array}$ & Entrevistas grupales & Reino Unido & $9-19$ & $N=108$ \\
\hline Bryce \& Fraser 41 & $\begin{array}{c}\text { Analizar la comprensión } \\
\text { sobre los riesgos } \\
\text { asociados a la exposición } \\
\text { de información personal } \\
\text { y el contacto con } \\
\text { desconocidos }\end{array}$ & Entrevistas grupales & Reino Unido & $9-19$ & $N=108$ \\
\hline Connolly 43 & $\begin{array}{l}\text { Analizar las razones } \\
\text { de adolescentes para } \\
\text { no reportar casos de } \\
\text { ciberbullying }\end{array}$ & Entrevistas grupales & Irlanda & $13-17$ & $N=59$ \\
\hline DeSmet et al. 42 & $\begin{array}{c}\text { Analizar los } \\
\text { comportamientos de } \\
\text { espectadores y sus } \\
\text { determinaciones para } \\
\text { actuar }\end{array}$ & Entrevistas grupales & Bélgica & $12-16$ & $\begin{array}{c}\mathrm{N}=61 \text { (32 mujeres, } \\
29 \text { varones) }\end{array}$ \\
\hline DeSmet et al. 68 & $\begin{array}{c}\text { Analizar las } \\
\text { determinaciones de } \\
\text { los espectadores para } \\
\text { defender a víctimas }\end{array}$ & Entrevistas grupales & Bélgica & $12-15$ & $\begin{array}{c}\mathrm{N}=33 \text { (11 mujeres, } \\
12 \text { varones })\end{array}$ \\
\hline Dredge et al. 48 & $\begin{array}{l}\text { Identificar los factores } \\
\text { que intervienen en el } \\
\text { impacto de ciberbullying } \\
\text { en víctimas }\end{array}$ & $\begin{array}{l}\text { Entrevistas } \\
\text { individuales }\end{array}$ & Australia & $15-24$ & $\begin{array}{c}\mathrm{N}=25 \text { (17 mujeres, } \\
8 \text { varones })\end{array}$ \\
\hline
\end{tabular}

(continúa) 
Cuadro 1 (continuación)

\begin{tabular}{|c|c|c|c|c|c|}
\hline \multirow[t]{2}{*}{ Autores } & \multirow[t]{2}{*}{ Objetivo } & \multirow[t]{2}{*}{ Metodología } & \multirow[t]{2}{*}{ País } & \multicolumn{2}{|c|}{ Muestra } \\
\hline & & & & Edad (años) & Tamaño \\
\hline Ging \& O'Higgins 56 & $\begin{array}{c}\text { Analizar cómo } \\
\text { las adolescentes } \\
\text { comprenden y } \\
\text { experimentan la } \\
\text { amistad, el conflicto } \\
\text { y el ciberbullying en } \\
\text { Facebook }\end{array}$ & $\begin{array}{l}\text { Entrevistas } \\
\text { individuales }\end{array}$ & Irlanda & $14-17$ & $\mathrm{~N}=26$ (mujeres) \\
\hline Keipi \& Oksanen 53 & $\begin{array}{l}\text { Analizar cómo } \\
\text { adolescentes } \\
\text { comprenden y } \\
\text { reaccionan ante los } \\
\text { riesgos en Internet }\end{array}$ & Narrativas escritas & Finlandia & $14-18$ & $\begin{array}{c}\mathrm{N}=258 \text { (143 mujeres, } \\
115 \text { varones })\end{array}$ \\
\hline Law et al. 50 & $\begin{array}{l}\text { Analizar los motivos de } \\
\text { la agresión virtual, en } \\
\text { especial, analizar las } \\
\text { agresiones proactivas y } \\
\text { reactivas }\end{array}$ & $\begin{array}{l}\text { Entrevistas en } \\
\text { profundidad }\end{array}$ & Canadá & $10-18$ & $\begin{array}{c}\mathrm{N}=15 \text { (10 mujeres, } \\
5 \text { varones })\end{array}$ \\
\hline Maher 66 & $\begin{array}{c}\text { Describir cómo se } \\
\text { desarrollan prácticas } \\
\text { de ciberbullying en la } \\
\text { escuela }\end{array}$ & Etnografía & Australia & $11-12$ & $N=22$ \\
\hline Nilan et al. 49 & $\begin{array}{l}\text { Identificar patrones } \\
\text { de comportamientos } \\
\text { sociológicos en el } \\
\text { ciberbullying }\end{array}$ & $\begin{array}{l}\text { Entrevistas } \\
\text { individuales }\end{array}$ & Australia & $15-18$ & $\begin{array}{c}\mathrm{N}=10 \text { (5 mujeres, } \\
5 \text { varones })\end{array}$ \\
\hline O’Brien \& Moules 63 & $\begin{array}{c}\text { Describir las } \\
\text { percepciones de víctimas } \\
\text { sobre el impacto que } \\
\text { tuvo en su vida el } \\
\text { ciberbullying }\end{array}$ & Entrevistas grupales & Reino Unido & $10-18$ & $\begin{array}{c}\mathrm{N}=17 \text { (14 mujeres, } \\
3 \text { varones })\end{array}$ \\
\hline Pabian et al. 61 & $\begin{array}{c}\text { Identificar las diferencias } \\
\text { y similitudes entre las } \\
\text { interacciones negativas } \\
\text { presenciales y virtuales }\end{array}$ & $\begin{array}{l}\text { Entrevistas } \\
\text { individuales }\end{array}$ & Bélgica & 13-14 & $N=34$ \\
\hline Parris et al. 59 & $\begin{array}{c}\text { Describir cómo los } \\
\text { adolescentes previenen } \\
\text { el ciberbullying }\end{array}$ & $\begin{array}{l}\text { Entrevistas } \\
\text { individuales }\end{array}$ & EE.UU. & $15-19$ & $N=40$ \\
\hline Parris et al. 60 & $\begin{array}{c}\text { Identificar las estrategias } \\
\text { para superar casos de } \\
\text { ciberbullying }\end{array}$ & $\begin{array}{l}\text { Entrevistas } \\
\text { individuales }\end{array}$ & EE.UU. & $15-19$ & $\begin{array}{c}\mathrm{N}=20 \text { (7 mujeres, } \\
13 \text { varones })\end{array}$ \\
\hline Patterson et al. 44 & $\begin{array}{l}\text { Comprender las } \\
\text { percepciones y } \\
\text { respuestas de } \\
\text { espectadores }\end{array}$ & $\begin{array}{l}\text { Entrevistas } \\
\text { individuales }\end{array}$ & Australia & $13-16$ & $\begin{array}{c}\mathrm{N}=24 \text { (13 mujeres, } \\
11 \text { varones) }\end{array}$ \\
\hline Pelfrey \& Weber 62 & $\begin{array}{l}\text { Comprender las } \\
\text { estrategias de prevención } \\
\text { y respuesta ante casos } \\
\text { de ciberbullying }\end{array}$ & Entrevistas grupales & EE.UU. & $11-14$ & $\begin{array}{c}\mathrm{N}=24 \text { (16 mujeres, } \\
8 \text { varones })\end{array}$ \\
\hline
\end{tabular}

(continúa) 
Cuadro 1 (continuación)

\begin{tabular}{|c|c|c|c|c|c|}
\hline \multirow[t]{2}{*}{ Autores } & \multirow[t]{2}{*}{ Objetivo } & \multirow[t]{2}{*}{ Metodología } & \multirow[t]{2}{*}{ País } & \multicolumn{2}{|c|}{ Muestra } \\
\hline & & & & Edad (años) & Tamaño \\
\hline Price et al. 28 & $\begin{array}{c}\text { Analizar las percepciones } \\
\text { de adolescentes sobre } \\
\text { espectadores }\end{array}$ & $\begin{array}{c}\text { Encuestas con } \\
\text { preguntas abiertas }\end{array}$ & EE.UU. & $M=15$ & $\begin{array}{c}\mathrm{N}=961 \text { (443 mujeres, } \\
481 \text { varones) }\end{array}$ \\
\hline Radovic et al. 55 & $\begin{array}{l}\text { Identificar los usos } \\
\text { de las redes sociales } \\
\text { en adolescentes con } \\
\text { depresión }\end{array}$ & $\begin{array}{l}\text { Entrevistas } \\
\text { individuales }\end{array}$ & EE.UU. & $13-20$ & $\begin{array}{c}N=23 \text { (18 mujeres, } \\
5 \text { varones })\end{array}$ \\
\hline Reason et al. 29 & $\begin{array}{c}\text { Analizar las experiencias } \\
\text { de víctimas y las maneras } \\
\text { en que lo sobrellevaron }\end{array}$ & $\begin{array}{c}\text { Entrevistas grupales e } \\
\text { individuales }\end{array}$ & EE.UU. & 18 & $\begin{array}{c}\mathrm{N}=8 \text { (4 mujeres, } \\
4 \text { varones) }\end{array}$ \\
\hline Samoh et al. 58 & $\begin{array}{c}\text { Analizar las percepciones } \\
\text { sobre la definición, } \\
\text { causas, consecuencias } \\
\text { y formas de abordar el } \\
\text { ciberbullying }\end{array}$ & $\begin{array}{c}\text { Entrevistas grupales e } \\
\text { individuales }\end{array}$ & Tailandia & $15-24$ & $N=136$ \\
\hline Ševčíková et al. 64 & $\begin{array}{c}\text { Describir cómo las } \\
\text { víctimas de ciberbullying } \\
\text { perciben la agresión } \\
\text { y en qué contextos es } \\
\text { considerada más grave }\end{array}$ & $\begin{array}{l}\text { Entrevistas } \\
\text { individuales }\end{array}$ & República Checa & $15-17$ & $\begin{array}{c}\mathrm{N}=16 \text { (7 mujeres, } \\
9 \text { varones })\end{array}$ \\
\hline Šléglová \& Černá 57 & $\begin{array}{c}\text { Describir cómo las } \\
\text { víctimas de ciberbullying } \\
\text { perciben la agresión y } \\
\text { qué estrategias utilizan } \\
\text { para superar la agresión }\end{array}$ & $\begin{array}{l}\text { Entrevistas } \\
\text { individuales }\end{array}$ & República Checa & $14-18$ & $\begin{array}{c}\mathrm{N}=15 \text { (13 mujeres, } \\
2 \text { varones })\end{array}$ \\
\hline Stacey 45 & $\begin{array}{l}\text { Analizar los usos } \\
\text { de las tecnologías } \\
\text { en adolescentes y } \\
\text { sus experiencias de } \\
\text { ciberbullying }\end{array}$ & Entrevistas grupales & Australia & $10-17$ & $N=74$ \\
\hline Vandebosch \& van Cleemput 67 & $\begin{array}{l}\text { Describir experiencias } \\
\text { y perspectivas de } \\
\text { ciberbullying }\end{array}$ & Entrevistas grupales & Bélgica & $10-19$ & $\begin{array}{c}\mathrm{N}=279 \text { (137 mujeres, } \\
142 \text { varones })\end{array}$ \\
\hline Varjas et al. 46 & $\begin{array}{l}\text { Describir experiencias } \\
\text { y perspectivas de } \\
\text { ciberbullying de } \\
\text { adolescentes de la } \\
\text { comunidad LGBTQ }\end{array}$ & $\begin{array}{l}\text { Entrevistas } \\
\text { individuales }\end{array}$ & EE.UU. & $15-18$ & $\begin{array}{c}\mathrm{N}=18 \text { (5 mujeres, } \\
13 \text { varones })\end{array}$ \\
\hline Varjas et al. 47 & $\begin{array}{c}\text { Describir las perspectivas } \\
\text { de adolescentes } \\
\text { sobre los motivos del } \\
\text { ciberbullying }\end{array}$ & $\begin{array}{l}\text { Entrevistas } \\
\text { individuales }\end{array}$ & EE.UU. & $15-19$ & $\begin{array}{c}\mathrm{N}=20 \text { (7 mujeres, } \\
13 \text { hombres) }\end{array}$ \\
\hline Wright 65 & $\begin{array}{c}\text { Analizar las } \\
\text { características de } \\
\text { las víctimas de } \\
\text { ciberbullying, sus } \\
\text { respuestas emocionales } \\
\text { y estrategias de } \\
\text { superación }\end{array}$ & $\begin{array}{l}\text { Entrevistas } \\
\text { individuales }\end{array}$ & EE.UU. & $12-14$ & $\begin{array}{c}\mathrm{N}=76 \text { (39 mujeres, } \\
37 \text { varones) }\end{array}$ \\
\hline
\end{tabular}

Fuente: elaboración própria. 
Cuadro 2

Análisis del corpus documental a partir de Critical Appraisal Skills Programme (CASP).

\begin{tabular}{|c|c|c|c|c|c|c|c|c|c|c|}
\hline \multirow[t]{2}{*}{ Estudios } & \multicolumn{10}{|c|}{ Ítems CASP * } \\
\hline & 1 & 2 & 3 & 4 & 5 & 6 & 7 & 8 & 9 & 10 \\
\hline Baas et al. 54 & $\mathrm{Si}$ & $\mathrm{Si}$ & Si & Si & $\mathrm{Si}$ & No & $\mathrm{Si}$ & $\mathrm{Si}$ & Si & $\mathrm{Si}$ \\
\hline Berne et al. 27 & $\mathrm{Si}$ & $\mathrm{Si}$ & Si & $\mathrm{Si}$ & $\mathrm{Si}$ & $\mathrm{Si}$ & $\mathrm{Si}$ & $\mathrm{Si}$ & $\mathrm{Si}$ & $\mathrm{Si}$ \\
\hline Betts \& Spenser 51 & Si & $\mathrm{Si}$ & Si & Si & Si & Si & Si & Si & Si & Si \\
\hline Bowler et al. 52 & Si & $\mathrm{Si}$ & Si & Si & Si & Si & Si & Si & Si & Si \\
\hline Brandau \& Evanson 26 & $\mathrm{Si}$ & $\mathrm{Si}$ & Si & $\mathrm{Si}$ & $\mathrm{Si}$ & $\mathrm{Si}$ & $\mathrm{Si}$ & $\mathrm{Si}$ & Si & $\mathrm{Si}$ \\
\hline Bryce \& Fraser 40 & $\mathrm{Si}$ & $\mathrm{Si}$ & Si & $\mathrm{Si}$ & $\mathrm{Si}$ & $\mathrm{Si}$ & $\mathrm{Si}$ & $\mathrm{Si}$ & $\mathrm{Si}$ & $\mathrm{Si}$ \\
\hline Bryce \& Fraser 41 & $\mathrm{Si}$ & $\mathrm{Si}$ & Si & Si & $\mathrm{Si}$ & $\mathrm{Si}$ & $\mathrm{Si}$ & $\mathrm{Si}$ & Si & $\mathrm{Si}$ \\
\hline Connolly 43 & $\mathrm{Si}$ & $\mathrm{Si}$ & Si & Si & $\mathrm{Si}$ & Si & $\mathrm{Si}$ & $\mathrm{Si}$ & Si & $\mathrm{Si}$ \\
\hline DeSmet et al. 42 & Si & $\mathrm{Si}$ & Si & Si & Si & No & Si & Si & Si & Si \\
\hline DeSmet et al. 68 & $\mathrm{Si}$ & $\mathrm{Si}$ & $\mathrm{Si}$ & $\mathrm{Si}$ & $\mathrm{Si}$ & $\mathrm{Si}$ & $\mathrm{Si}$ & $\mathrm{Si}$ & $\mathrm{Si}$ & $\mathrm{Si}$ \\
\hline Dredge et al. 48 & Si & $\mathrm{Si}$ & Si & Si & Si & No & No & Si & Si & Si \\
\hline Ging \& O'Higgins 56 & $\mathrm{Si}$ & $\mathrm{Si}$ & Si & Si & $\mathrm{Si}$ & $\mathrm{Si}$ & $\mathrm{Si}$ & $\mathrm{Si}$ & Si & $\mathrm{Si}$ \\
\hline Keipi \& Oksanen 53 & $\mathrm{Si}$ & $\mathrm{Si}$ & Si & $\mathrm{Si}$ & $\mathrm{Si}$ & $\mathrm{Si}$ & $\mathrm{Si}$ & $\mathrm{Si}$ & $\mathrm{Si}$ & $\mathrm{Si}$ \\
\hline Law et al. 50 & Si & $\mathrm{Si}$ & Si & Si & Si & No & Si & Si & Si & Si \\
\hline Maher 66 & Si & $\mathrm{Si}$ & Si & Si & Si & Si & Si & Si & Si & Si \\
\hline Nilan et al. 49 & Si & $\mathrm{Si}$ & Si & Si & Si & $\mathrm{Si}$ & No & Si & Si & Si \\
\hline O'Brien \& Moules 63 & $\mathrm{Si}$ & $\mathrm{Si}$ & $\mathrm{Si}$ & $\mathrm{Si}$ & $\mathrm{Si}$ & No & $\mathrm{Si}$ & $\mathrm{Si}$ & $\mathrm{Si}$ & $\mathrm{Si}$ \\
\hline Pabian et al. 61 & $\mathrm{Si}$ & $\mathrm{Si}$ & Si & $\mathrm{Si}$ & $\mathrm{Si}$ & $\mathrm{Si}$ & $\mathrm{Si}$ & $\mathrm{Si}$ & Si & $\mathrm{Si}$ \\
\hline Parris et al. 59 & Si & $\mathrm{Si}$ & Si & Si & Si & $\mathrm{Si}$ & Si & Si & Si & Si \\
\hline Parris et al. 60 & $\mathrm{Si}$ & $\mathrm{Si}$ & Si & Si & $\mathrm{Si}$ & $\mathrm{Si}$ & $\mathrm{Si}$ & $\mathrm{Si}$ & Si & $\mathrm{Si}$ \\
\hline Patterson et al. 44 & Si & $\mathrm{Si}$ & Si & Si & $\mathrm{Si}$ & Si & Si & Si & Si & Si \\
\hline Pelfrey \& Weber 62 & $\mathrm{Si}$ & $\mathrm{Si}$ & $\mathrm{Si}$ & $\mathrm{Si}$ & $\mathrm{Si}$ & $\mathrm{Si}$ & $\mathrm{Si}$ & $\mathrm{Si}$ & $\mathrm{Si}$ & $\mathrm{Si}$ \\
\hline Price et al. 28 & Si & $\mathrm{Si}$ & Si & Si & $\mathrm{Si}$ & Si & $\mathrm{Si}$ & $\mathrm{Si}$ & Si & $\mathrm{Si}$ \\
\hline Radovic et al. 55 & Si & $\mathrm{Si}$ & Si & Si & $\mathrm{Si}$ & $\mathrm{Si}$ & $\mathrm{Si}$ & Si & Si & $\mathrm{Si}$ \\
\hline Reason et al. 29 & $\mathrm{Si}$ & $\mathrm{Si}$ & Si & Si & $\mathrm{Si}$ & $\mathrm{Si}$ & $\mathrm{Si}$ & $\mathrm{Si}$ & Si & $\mathrm{Si}$ \\
\hline Samoh et al. 58 & Si & $\mathrm{Si}$ & Si & Si & Si & $\mathrm{Si}$ & Si & Si & Si & Si \\
\hline Ševčíková et al. 64 & $\mathrm{Si}$ & $\mathrm{Si}$ & $\mathrm{Si}$ & $\mathrm{Si}$ & $\mathrm{Si}$ & $\mathrm{Si}$ & $\mathrm{Si}$ & $\mathrm{Si}$ & $\mathrm{Si}$ & $\mathrm{Si}$ \\
\hline Šléglová \& Černá 57 & $\mathrm{Si}$ & $\mathrm{Si}$ & $\mathrm{Si}$ & $\mathrm{Si}$ & $\mathrm{Si}$ & $\mathrm{Si}$ & $\mathrm{Si}$ & $\mathrm{Si}$ & $\mathrm{Si}$ & $\mathrm{Si}$ \\
\hline Stacey 45 & $\mathrm{Si}$ & $\mathrm{Si}$ & $\mathrm{Si}$ & $\mathrm{Si}$ & $\mathrm{Si}$ & No & No & $\mathrm{Si}$ & $\mathrm{Si}$ & $\mathrm{Si}$ \\
\hline Vandebosch et al. 67 & $\mathrm{Si}$ & $\mathrm{Si}$ & Si & Si & $\mathrm{Si}$ & $\mathrm{Si}$ & No & $\mathrm{Si}$ & Si & $\mathrm{Si}$ \\
\hline Varjas et al. 46 & $\mathrm{Si}$ & $\mathrm{Si}$ & Si & Si & Si & No & Si & $\mathrm{Si}$ & Si & Si \\
\hline Varjas et al. 47 & $\mathrm{Si}$ & $\mathrm{Si}$ & $\mathrm{Si}$ & $\mathrm{Si}$ & $\mathrm{Si}$ & $\mathrm{Si}$ & $\mathrm{Si}$ & Si & $\mathrm{Si}$ & $\mathrm{Si}$ \\
\hline Wright 65 & $\mathrm{Si}$ & $\mathrm{Si}$ & Si & Si & $\mathrm{Si}$ & $\mathrm{Si}$ & $\mathrm{Si}$ & $\mathrm{Si}$ & Si & $\mathrm{Si}$ \\
\hline
\end{tabular}

Fuente: elaboración propia a partir de Critical Appraisal Skills Programme (CASP. https://casp-uk.net/wp-content/ uploads/2018/03/CASP-Qualitative-Checklist-2018_fillable_form.pdf).

* 1 = ¿Hubo una declaración clara de los objetivos de la investigación?; 2 = ¿Es apropiada una metodología cualitativa?; 3 = ¿Fue el diseño de investigación apropiado para dirigir los objetivos de la investigación?; 4 = ¿Fue la estrategia de reclutamiento adecuada a los objetivos de la investigación?; 5 = ¿Se recopilaron los datos de una manera que abordara el problema de la investigación?; 6 = ¿Se ha considerado adecuadamente la relación entre el investigador y los participantes?; 7 = ¿Se han tenido en cuenta las cuestiones éticas?; 8 = ¿Fue el análisis de datos suficientemente riguroso?; 9 = ¿Hay una declaración clara de los resultados?; 10 = ¿Qué tan valiosa es la investigación?. 


\section{Resultados}

Los adolescentes relatan que, indistintamente de su género, pueden asumir tres roles en el ciberbullying: víctimas, perpetradores, o espectadores $26,27,28,29,40,41,42,43,44,45,46,47,48,49,50,51,52,53,54,55,56,57$, $58,59,60,61,62,63,64,65,66,67,68$. Al hablar de sus experiencias personales y/o la de sus pares, no hablan de los roles como un "estado permanente", sino como una posición situacional que se ocupa 40,50,52. De este modo, las mismas personas pueden ser agresoras y agredidas a la vez en diferentes escenarios $42,43,49,50,52,63,68$. También es variado el papel desempeñado por los espectadores. Ellos pueden reforzar la agresión apoyando a los perpetradores, defender a la víctima, o solo observar 41,42,44,45,48,50,51,52,68. Si bien en la práctica del ciberbullying puede que haya posiciones recurrentes entre quienes ejercen roles de víctimas, perpetradores y espectadores, para los adolescentes, la comunicación mediada por las tecnologías hace que sea difícil discernirlos al observar una agresión. Los adolescentes desconocen su contextualización, cuál es la trayectoria de lo sucedido y si proviene o continua de modo paralelo en el espacio no virtual. En parte, esta ambivalencia y alternancia se da porque la articulación entre los espacios virtual y presencial es constante, y en ellos las personas ocupan diferentes posiciones $42,43,46,49,52,67,68$. Las agresiones adquieren una dinámica compleja y generan la percepción de que nunca culminan, debido a los movimientos entre los espacios y la participación de más actores 26,28,52,61.

\section{Los adolescentes como agresores: qué hacen y por qué}

En todas las expresiones del ciberbullying se observa que los adolescentes son expuestos a situaciones de humillación, en las que se daña su imagen y valoración social 26,27,28,29,40,41,42,43,44,45,46,47,48,49,50,51, $52,53,54,55,56,57,58,59,60,61,62,63,64,65,66,67,68$. Los modos más frecuentes en los que se emplea son criticar o insultar 27,29,42,43,44,45,46,49,50,51,52,53,54,56,57,61,64,65,67,68; compartir información personal, fotos y videos (manipuladas o no con programas de edición) 27,29,40,43,44,45,46,48,50,51,52,57,58,64,67; amenazar 42,44,45,46, 50,51,52,54,57,61,65,67,68; hackear cuentas personales para enviar fotos humillantes o escribir comentarios que perjudiquen los vínculos sociales del propietario de esa cuenta $27,45,49,51,53,54,58,61,65,67$; crear páginas específicas para divulgar información, imágenes o críticas sobre alguien 45,46,50,57,58,64,67; y excluir de grupos y actividades virtuales 43,45,46,49,50,51,56,58,61,63,67.

En los relatos identificamos dos motivos principales que desencadenan el ciberbullying. El primero se relaciona con la sanción a quienes no cumplen con los estándares de comportamiento y belleza. Los adolescentes a través del ciberbullying puntualizan en sus pares aspectos que son sensibles para sentirse reconocidos, sobre todo atributos que no cumplen con los estándares sociales de comportamiento $26,27,42,43,47,49,50,52,54,56,61,67,68$, de producción estética $26,27,43,47,49,50,52,53,54,56,58,62$ y orientación sexual $26,27,29,46,49,54,56,64$. Se agreden a aquellas personas por ser distintas, y por lo que los otros piensan de ellas, en función de los modos de ser establecidos socialmente 26,27,29,42,43,49,54,56,65, 67,68. En los relatos, los adolescentes le otorgan cierta responsabilidad a las víctimas por ser agredidas, por considerar que ellas son las que deciden ser diferentes o las que hicieron algo que originara esa agresión 27,29,42,50,56,58,61,68.

Si bien transcurre en el espacio virtual, las agresiones suelen dirigirse a personas que conocen presencialmente $26,47,49,64,65$. A través de esas prácticas los adolescentes buscan construir pertenencia

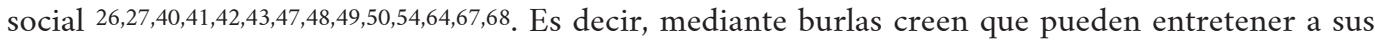
pares, construir vínculos y obtener un reconocimiento que les permita posicionarse socialmente 27,40, $41,42,43,47,48,49,50,64,67,68$. La valoración es otorgada por los espectadores que aprueban esa acción y se expresa en la cantidad de "me gusta" -likes u otros dispositivos que indican aprobación en las redesque reciben en sus publicaciones $27,41,47,49,53,56$. Esta estrategia es más fácil de implementar, cuando las agresiones se dirigen hacia una persona que ya cuenta con una valoración negativa por parte de sus pares, porque es más probable que los espectadores legitimen la agresión y la víctima no cuente con vínculos interpersonales fuertes que puedan defenderla 26,49,54,56,65. También intentan elegir como espectadores a miembros de su grupo social de pertenencia que apoyen la agresión 47,49,50,52,64,65.

El segundo motivo del ciberbullying identificado es la venganza 26,43,46,47,48,49,50,51,52,54,58,61,65,67. En este caso, los adolescentes agreden a personas que les han generado algún tipo de ofensa, especialmente en discusiones 26,29,43,46,47,48,49,50,51,52,54,56,58,61,65,67. Entre las situaciones que dan origen a una 
venganza, comentan que es usual que adolescentes que sufrieron ciberbullying y/o bullying escolar, reaccionen con nuevas agresiones hacia sus agresores 43,47,49,50,52,54,65,67. Describen a las víctimas frecuentes de bullying escolar como personas raras, tímidas y pequeñas 42,47,49,50,65,67. En los espacios presenciales, estas características físicas expresan desigualdades de poder que no están en la virtualidad 42,47,49,50,67,68. Entienden así, que las TICs empoderan a estas personas para poder responder a sus perpetradores con agresiones 47,49,50,67. Cuando eso ocurre, consideran justificado su comportamiento, es decir, como víctimas es legítimo actuar en pos de su defensa con otras agresiones 43,50. En este sentido, la virtualidad se percibe como una herramienta que permite a quienes fueron víctimas de agresiones en el espacio presencial, agredir en el espacio virtual 47,49,50,67.

\section{Los adolescentes como víctimas: vivencias y reacciones ante las agresiones}

Para los adolescentes el ciberbullying se vive como una agresión que no finaliza en el acto, porque persiste en el espacio virtual y en la memoria de la audiencia potencial 29,40,42,43,44,48,51,52,54,58,63,65,68. En un espacio donde la imagen representa un medio para expresar su identidad y valoración, las prácticas dirigidas a difundir fotos y vídeos son percibidas como más dañinas que las agresiones escritas o físicas 42,43,44,54,55,58,68. Así, la simple presencia de una audiencia agrava los actos, porque este tipo de violencia deja una marca en su identidad virtual de forma permanente 29,40,43,44,48,51,55,58,65. Esas condiciones hacen que la repetición posible de la agresión pase a un segundo plano y consideren que un solo acto es suficiente para sentirse humillados y lastimados 49,54,58.

Ante agresiones las víctimas sienten impotencia, porque no pueden controlar la participación de los espectadores, eliminar el material audiovisual para detener su difusión 29,48,49,54,57,64, ni escapar de la situación, es decir, no pueden detener el envío de mensajes que reciben 26,29,46,49,51,52,57,60,65. A la vez, el malestar se agrava si los agresores actúan desde el anonimato, por no comprender quién y por qué los agrede 26,29,40,43,48,49,54,65,67.

Es usual que traduzcan el enojo que sienten por la humillación y la impotencia en agresiones hacia sus perpetradores mediante insultos, difusión de rumores o creación de páginas web específicas para denunciarlos 29,43,47,49,50,52,54,57,58,65,67. Otra manera de reaccionar consiste en minimizar el ciberbullying al tomarlo como una práctica habitual o una broma, que puede sucederle a toda persona que participa en ese espacio 27,45,48,51,56,58,60,61. Explican que actuar como si no les hubiera afectado evita que se diseminen rumores sobre eso, y demuestra que no era algo para mantener en privado, es decir, que no era objeto de vergüenza o de ocultamiento 45,61 .

A pesar de la vulnerabilidad en la que se encuentran, los adolescentes ante una agresión destacan su deseo y voluntad de controlar en forma autónoma la situación que atraviesan 41,43,44,45,52,54,56,58,60. Este posicionamiento se observa en su resistencia a conversar sus experiencias con adultos 26,29,43,44,45, $52,54,56,57,58,60,61$. En general, perciben que estos no comprenden el espacio virtual, sus valores y prácticas, y hacen que sus reacciones les ocasionen nuevos problemas a enfrentar 26,29,43,44,45,52,54,56,57,62. Por un lado, se refieren a la posibilidad de vivenciar otros procesos de victimización, a partir de las denuncias de adultos responsables a las autoridades escolares. Los adolescentes traducen los mecanismos escolares de resolución de conflictos como situaciones de exposición en las que los agredidos se sienten exhibidos 42,43,44,45,51,52,54,60,62,68. Así, los agresores se darían cuenta que fueron denunciados y podrían desencadenarse nuevos actos de agresión y aislamiento social 42,43,52,54,60,62,68. El segundo escenario se relaciona con el control de los adultos sobre la sociabilidad virtual adolescente. Cuando intervienen, algunas de sus decisiones se orientan a restringir el acceso a los dispositivos electrónicos y/o a supervisar el comportamiento en las redes sociales, con el fin de evitar nuevos episodios de agresiones 29,42,43,44,45,52,54,58,59,60,62. Sin embargo, la posibilidad de quedar por fuera de su sociabilidad virtual es algo que desean evitar, porque les implica aislarse socialmente 29,43,54,58. Por estas razones, hablarían con adultos solo si sienten que no pueden resolver la situación y temen por su seguridad $43,44,45,56,58,59,60,61,62$. En caso contrario, prefieren buscar contención y pedir consejos a sus pares o hermanos mayores que comprenden las experiencias por ser parte del espacio virtual 29,40,43,44,45,53,54,57, $58,59,60,61,62$. Las adolescentes son más proclives a pedir ayuda que los varones. Ellos son más reticentes, incluso a conversar con sus pares, porque esto podría ser visto como una señal de debilidad. Sienten que deben aparentar ser fuertes y una forma de serlo es manejar la situación solos o confrontar físicamente a los agresores 26,27,43,49,52,54,65. 
Si bien los adolescentes cuando son agredidos sostienen su sociabilidad virtual, suelen modificar los modos de usar las tecnologías 27,41,43,49,51,52,55,56,57,60. Principalmente, reducen la exposición personal de información y fotos $27,41,43,49,51,52,55,56,60$, aprenden técnicas de seguridad de sus cuentas y son más precavidos en sus interacciones $55,57,59$.

\section{Los adolescentes como espectadores y sus motivos para participar}

Los adolescentes expresan que ante un hecho de agresión su intervención como espectadores depende de aspectos contextuales, como son los vínculos que guardan con las personas agredidas y agresoras, el tipo de agresión y el género de quienes se involucran 28,42,44,45,52,62,67. Una de las razones, por las que toman en cuenta tantas variables, se debe a que para ellos defender a la persona que ha sido agredida implica también exponerse a ser objeto de represalias por parte del perpetrador inicial o de otras personas 26,28,42,43,44,52,56,62,68.

Antes de decidir intervenir, mencionan la necesidad de recuperar la mayor cantidad posible de información sobre los hechos sucedidos. El riesgo de acusar o defender a partir de una malinterpretación sería justificativo para ser criticado por sus pares $42,44,45,62,68$. Esto se debe a que acceden a escenas que son parte de un proceso que desconocen, así agresiones aparentes pueden en realidad ser bromas entre conocidos 40,42,43,44,45,49,54,67,68 o ignorar si los protagonistas fueron perpetradores o víctimas $42,43,50,52,68$. Cuando los espectadores conocen a los protagonistas, les es sencillo recuperar información, pero cuando no lo son, prefieren no intervenir ante la falta de esta 42,44,62,68.

En general, refieren actuar a favor de las personas agredidas cuando estas son sus amigos 28,42,44,61,62,68. Aún si prefieren no participar por miedo a ser agredidos, sienten que tienen una obligación, ya que les afecta más ser penado socialmente por no respetar los valores de proximidad que ser objeto de agresión 28,44,44,62,68. Como suelen ser los grupos de pertenencia quienes actúan a favor, los adolescentes que no pertenecen a ninguno no tienen pares que intervengan en su defensa o contención, encontrándose en una posición de mayor vulnerabilidad 42,44,49,50,65,68. No obstante, ciertos adolescentes suelen actuar a pesar de no tener un vínculo. Lo hacen motivados por sentir empatía al haber sido ellos mismos víctimas en otra ocasión o por percibirse con suficiente poder para posicionarse como referentes 28,42,44,52. En este sentido, también entra en juego la valoración social de los espectadores 42,44,52. De este modo, cuando tienen una apreciación social buena -definida como popular o fuerte- se espera que actúen a favor de su amigo y son criticados cuando deciden no hacerlo 28,42. En cambio, los espectadores que no poseen esa valoración, no se sienten seguros para intervenir por el miedo de ser también agredidos $28,42,43,44,52,54$.

Para evitar acciones que los expondrían a nuevos procesos de victimización, los adolescentes refieren que participarían más en la defensa del agredido si pudieran hacerlo anónimamente 28,52,53. Una de las estrategias que tienen en cuenta es aproximarse a agresores y agredidos de manera independiente y privada para no avergonzarlos y provocarlos en público 42,52. También tienen en cuenta la valoración de la agresión ocurrida. Suelen no intervenir cuando la interpretan como una broma irónica entre amigos 40,42,43,44,45,49,54,67. Por el contrario, se legitima la intervención en la medida en que la frecuencia o el tipo de agresión aumenta 28,42,44,58,62,67. Su repetición, las amenazas físicas y la percepción de que las personas no pueden defenderse por sí mismas motivan a intervenir 42,44,62. Por último, los espectadores tienen en cuenta el género para definir su actuación. Dicen que las adolescentes expresan mayor la afección que les provoca el ciberbullying y el intento de defenderse deriva en peleas extensas y complejas por intentar incluir a otras personas en la discusión, mientras que los varones se muestran más reservados o minimizan los efectos de la agresión en sus vidas 44,45,56,60. Por eso, cuando las víctimas son mujeres, intentan no involucrarse o hacerlo por mensajes privados 44 .

\section{Presentación de la síntesis: el ciberbullying como expresión de la sociabilidad virtual}

Goffman 32 sostiene que las personas en la sociabilidad cotidiana se comportan como actores que desempeñan un rol destinado a satisfacer ciertas expectativas que se depositan en ellas. En sus acciones suelen mostrarse de la manera en que esperan ser reconocidas, eligiendo algunos atributos personales y ocultando otros desacreditantes. De esta forma, la interacción implica siempre la representación de una fachada de sí ajustada a las expectativas sociales vinculadas con la posición, identidad e intencio- 
nes de cada actor. Podemos decir que las redes sociales actúan como la fachada donde los adolescentes están en constante presentación de sí mismos. El material personal que se publica se construye para la audiencia que compone el espacio virtual, y se pone a su disposición para que expresen qué piensan sobre él a través de comentarios, reproducciones y "me gusta". Así, en las redes sociales se desata una competencia para obtener reconocimiento que se traduce en un capital simbólico y social para quien lo alcanza 69. La importancia que adquiere en los adolescentes la obtención de reconocimiento se relaciona con el proceso de formación de su identidad, donde pueden definir quiénes son a partir de la devolución de los otros sobre sus publicaciones, porque el "sí mismo" (the self) que se construye es producto de esa interacción 32 .

Los adolescentes, según sus capitales, ocupan determinadas posiciones en su sociabilidad y entablan relaciones de poder atravesadas por expectativas de reconocimiento mutuo. En este contexto, consideramos al ciberbullying como uno de los mecanismos por el cual los adolescentes ordenan, producen y reproducen esas posiciones de poder dadas por el reconocimiento, a través de actos de identificación y diferenciación con sus pares $34,35,37$. Si se hace foco en las diversas prácticas con las que se lleva a cabo el ciberbullying, observamos que la característica que las atraviesa es que el objetivo es dañar la imagen y la valoración social de las personas. No es meramente la intención de "generar daño", es un daño en la identidad que se ejerce al asignarle una valoración negativa y crítica a aspectos del cuerpo, la producción estética, la personalidad y el comportamiento de las personas. En términos de Goffman 32, diremos que la agresión se dirige a desacreditar la fachada que muestran los adolescentes.

En el corpus de análisis identificamos dos motivos que desencadenan el ciberbullying. El primero se vincula con los modos en que se pone en juego el reconocimiento entre pares a partir de las fachadas construidas. Como destaca Goffman 32, en una actuación la audiencia cree en el papel que está cumpliendo, pero también evalúa y juzga si es adecuado a partir de sus expectativas de comportamiento. Al respecto, Butler 38 sostiene que la generización del cuerpo descansa en convenciones que sancionan y proscriben cómo cada uno actúa su propio cuerpo. Siguiendo a Butler 38, podemos decir que el ciberbullying también refleja la vigilancia y juicio sobre los actos de género. A través de él, los adolescentes sancionan aquellos atributos y comportamientos que transgreden los ideales que componen sus repertorios valorativos 70 . En la búsqueda de su identidad, los ideales operan como sus márgenes de referencia, como el punto al que se espera alcanzar. Así, se critican aquellos aspectos que se rechazan valorativamente, con los cuales no se sienten identificados. Por eso, se observa en los resultados que los adolescentes justifican que las agresiones sean dirigidas a estas personas. En algunos casos, responsabilizan a las víctimas por no comportarse como el deber ser y, en otras, si bien no están de acuerdo con la agresión (es decir, nos les parece que esté bien ni lo harían), sí entienden por qué les sucede.

El segundo motivo es la venganza, es decir, las agresiones que se emplean como la resolución de un conflicto previo para sancionar a personas que amenazaron sus expectativas de reconocimiento 36 . Cuando la valía de los adolescentes es puesta en duda, buscan su restitución con la agresión al desacreditar la imagen ante una audiencia. Gimenez ${ }^{34}$ sostiene que quienes ocupan posiciones dominadas aceptan la definición dominante de su identidad o se rebelan para poder disputar la escala de valores que las ubica en una posición devaluada. En estos casos, la virtualidad les ofrece un medio para resistir, porque la distancia física y el uso del anonimato los protege de continuar siendo victimizados y ofrecen las condiciones para que intenten cambiar las posiciones de reconocimiento en las que se ubican.

En los motivos observamos que opera la disputa por el reconocimiento, a través de un proceso de identificación por oposición por el cual yo soy lo que el otro no es. Como indica Goffman ${ }^{33}$, cuando se marca un atributo desacreditador en una persona -un estigma- también se confirma la normalidad de otro. De este modo, los adolescentes en el acto de la agresión establecen una oposición y se posicionan jerárquicamente ante otra persona invocando discursos dominantes 34,37. Si la agresión es legitimada por sus pares, quien agrede recupera para sí el valor de defender los repertorios valorativos dominantes y obtiene o sustenta capital social y simbólico. Mientras que las personas agredidas pierden ese capital y se ubican en un lugar de inferioridad y/o exclusión ante la mirada de los demás 34 . La agresión desde esta perspectiva no es estática ni fija posiciones. Debe pensarse como un proceso dinámico por el cual se disputa constantemente el reconocimiento de los pares, la afirmación de la identidad y las posiciones a ocupar en la sociabilidad. Por eso en el ciberbullying puede haber alternancia de roles. 
Quienes se encuentran en una posición de inferioridad en una escena, pueden intentar cambiar su posición en otra 34 .

Con respecto a los espectadores, observamos que, cuando apoyan la agresión, se asumen como portadores de estándares sociales que defienden y buscan su reconocimiento por eso. Sin embargo, cuando no apoyan la agresión, en lo posible prefieren no involucrarse, porque los expone también a ser agredidos. Esto sucede cuando confunden un ataque con una broma entre amigos. Si intervienen pensando que es una agresión, la respuesta de los protagonistas no solo será crítica, sino que también expresará que no pertenecen al grupo en el que se entiende el sentido de la broma. Es decir, no comprender es equivalente a no pertenecer y eso implica para ellos visibilizar su posición en la configuración de las relaciones. Al intervenir, se ponen en juego sus capitales sociales y simbólicos, tanto por las reacciones de los agresores y agredidos, como por el juicio de la audiencia una vez que la acción queda inscripta en el espacio virtual. Por eso, mencionan que están más dispuestos a participar de manera privada, esto es, entrando en contacto presencialmente o por chat privado con las personas agresoras o agredidas. Así, logran intervenir sin comprometer ante la audiencia sus capitales y su posición en el marco de la sociabilidad.

Las variables que sí los interpelan a intervenir públicamente son la pertenencia a grupos y el tipo de agresión. Se espera que quienes integran el grupo del agredido actúen en su defensa. Si la ayuda es por medio de canales privados, las víctimas pueden sentirse acompañadas en sus vínculos próximos, aunque esto no elimina el daño que produce la exposición pública del material que atenta contra su identidad. Por eso, el deseo de quienes son agredidos es que los pares intervengan públicamente para mostrar ante la audiencia que están acompañados por personas dispuestas a defenderlos. Si los pares no intervienen, otros espectadores pueden criticarlos por no cumplir con el deber que exige el vínculo que mantienen con la persona agredida. Con respecto a la gravedad de la agresión, en sus repertorios simbólicos las describen con diferentes grados de tolerancia. Algunas se encuentran naturalizadas por su frecuencia (por lo general insultos) o no las consideran lo suficientemente intolerables como para comprometer sus capitales en alguna acción. Cuando consideran que las agresiones son intolerables para sus repertorios, están más dispuestos a intervenir, incluso aunque la persona agredida no sea de su grupo de pertenencia.

Las personas agredidas en el ciberbullying son expuestas a situaciones de humillación ante una audiencia. Si la audiencia legitima la agresión, la impresión de sí de la víctima con la que esperaba ser valorada es desacreditada y alterada 32,33 . Los adolescentes sienten vergüenza, tristeza, inseguridad, depresión y aislamiento social por esta victimización. Dañar la valoración de las personas tiene implicaciones en sus formas de vincularse, porque altera su autoestima y su percepción de cuáles son las expectativas de los otros sobre ellas 32,33 . De esta manera, se modifica su posición social en la configuración de relaciones con sus pares. Como la agresión queda inscripta en el espacio virtual, sienten que el daño es permanente. La agresión puede inscribirse como un estigma, porque al conocer a nuevas personas, los adolescentes temen que estas hayan visto o puedan llegar a ver el material que daña su imagen 33. Goffman 32 destaca que las personas no se presentan de la misma manera ante todos los grupos sociales, porque tienen diferentes expectativas de comportamiento. La permanencia de la agresión impide que las personas puedan hacer uso de esa segmentación y control de sus impresiones, por lo que pierden la autonomía y seguridad en su interacción social. Así, la agresión hecha estigma las obliga a controlar la presentación de sus atributos personales con la premisa que los otros podrían identificarlas como desacreditables 33 .

Ante la lesión de reconocimiento, los adolescentes pueden buscar su restitución mediante diversas alternativas, no necesariamente excluyentes 36 . Pueden buscar apoyo y contención (reconocimiento) en su grupo de pertenencia, pueden ignorarlo y pretender que no fue lesionada su valía (como, por ejemplo, tomarlo como una broma usual), pueden responder a sus agresores y buscar reconocimiento en sus pares para sancionar el comportamiento agresor.

Si bien las víctimas disminuyen su participación en las redes sociales, no se alejan de ellas. Esto indica la importancia que tiene el espacio virtual en su sociabilidad. Pueden evitar publicar información para no exponerse a agresiones, pero continúan observando qué sucede y participando de una manera más precavida. El deseo de permanecer se relaciona con la importancia que tiene para ellos el sentimiento de pertenencia a grupos. Por eso, prefieren pedir contención en sus grupos de pares. También, se observa que es más usual que las mujeres pidan más ayuda que los varones. Los adoles- 
centes tienden más a soportar la agresión o responder con agresiones físicas. Esta actitud se vincula con el estereotipo de masculinidad que exige un comportamiento que demuestre valentía y fuerza 35.

Pensar el ciberbullying como una expresión singular de la sociabilidad virtual tiene el objetivo de reconocer, por un lado, los procesos de construcción de las identidades a través del reconocimiento brindado por las personas con las que se interactúa y, por el otro, las disputas por la obtención de ese reconocimiento y la configuración de relaciones de poder entre los adolescentes.

\section{Comentarios finales}

Una meta-etnografía está completa cuando logramos identificar en el conjunto de los textos analizados una síntesis, cuyo significado los contiene y, al mismo tiempo, los trasciende ${ }^{30}$. En este caso, el trabajo permitió sintetizar los hallazgos de 33 investigaciones que se complementan y se refuerzan, construyendo una línea argumentativa que otorga una visión más completa del ciberbullying desde los tres roles que suelen asumir sus protagonistas. Consideramos que los resultados alcanzados son significativos para el campo de la salud colectiva, porque ofrecen elementos teóricos para comprender esta violencia entre adolescentes, para diseñar y discutir políticas públicas en pos de su tratamiento, y para generar nuevos interrogantes, con el fin de profundizar el estudio del ciberbullying.

En los resultados observamos que la característica que reúne al conjunto de expresiones de ciberbullying es la intención de afectar la valoración social de las personas agredidas. Para analizarlo, consideramos la construcción de las identidades como un proceso constante que se da a partir del reconocimiento brindado por los otros. En este marco, propusimos que el ciberbullying se ejerce como un mecanismo de construcción de identidad, a partir de los procesos de identificación y oposición con pares. Es decir, al desacreditar a una persona por un atributo de su personalidad, producción estética, comportamiento y sexualidad, se marca una diferencia con esta y se demuestra una identificación con los atributos opuestos a los que se dirige la agresión. En segundo lugar, observamos que, en ese proceso, el ciberbullying a la vez reproduce y disputa posiciones de reconocimiento, porque al desacreditar a una persona ante una audiencia afecta sus fuentes de valoración social que sustentan esa posición. En tercer lugar, consideramos también que es una práctica punitiva de transgresiones a un orden sociosimbólico dominante, ya que se sanciona y legitima formas específicas de ser y estar. Por las razones expuestas, sostenemos que el ciberbullying no es una nueva violencia, sino una nueva expresión de tipos de violencias ya enraizadas en procesos de identificación, poder y género.

La propuesta de analizar el ciberbullying desde el interaccionismo simbólico y disputas por el reconocimiento es una alternativa innovadora en el campo de estudio. La mayoría de las investigaciones ofrecen explicaciones basadas en la construcción de perfiles epidemiológicos y modelos predictivos de comportamiento, a partir de teorías como la del estrés general, la desconexión moral, el comportamiento planificado y el efecto del espectador 5,14,16,18,19. Estas perspectivas tienden a individualizar y medicalizar las causas del ciberbullying y a otorgarle un papel central al espacio virtual (en especial al anonimato) como factor determinante, en vez de identificar cómo los adolescentes hacen uso de él y cómo el ciberbullying se entreteje en su sociabilidad.

En correspondencia a estas explicaciones predominantes, actualmente, las políticas para prevenir el ciberbullying en su mayoría se dirigen a brindar charlas sobre los "riesgos" de la exposición en Internet, recomendaciones sobre la protección de datos personales, y un fuerte incentivo de la supervisión parental de la sociabilidad virtual de sus hijos 24,25,71,72. Sin embargo, estas medidas de prevención parecen insuficientes o poco adecuadas. En parte, porque observamos que los adolescentes intentan ocultar sus experiencias de ciberbullying para evitar que sean controlados o aislados de la sociabilidad virtual, por lo que la participación de los adultos debe ser a partir de la comprensión del rol que cumple el espacio virtual en sus vidas y no desde la sanción o restricción. A la vez, porque no parece que los adolescentes desconocen los riesgos o los medios para protegerse, sino que se tratan de agresiones aprendidas y arraigadas en su sociabilidad por la búsqueda del reconocimiento y las relaciones de identificación y oposición con sus pares. Por lo tanto, brindar información sobre riesgos y consecuencias en la salud, incrementar la supervisión y reducir la participación, serían recomendaciones insuficientes para abordar el núcleo del problema. Más bien, consideramos que las políticas deberían orientarse a formas de producir reconocimiento entre los adolescentes que no 
sean a través de la diferencia y competencia con sus pares mediante la descalificación de su identidad. Destacamos, entonces, la necesidad de diseñar políticas incorporando la perspectiva de la población a la que se quiere dirigir.

Por último, la meta-etnografía permitió identificar patrones transversales en el ciberbullying, pero también lagunas de conocimiento sobre esta agresión. Si bien pudieron evidenciarse diferencias de género en los hallazgos de los artículos al describir prácticas y discursos de varones y mujeres, observamos que ninguno tomó la perspectiva de género como construcción metodológica y analítica. Así, consideramos que futuras investigaciones podrían orientarse a estudiar cómo se construye y expresa el ciberbullying como formas de producir identidades de género; realizar estudios de casos con la mirada de todas las personas involucradas para reconstruir el proceso de agresión; y analizar cómo las características de la sociabilidad virtual habilitan y legitiman esta violencia.

\section{Colaboradores}

C. Moretti participó en la concepción y diseño del estudio, en la conformación del corpus, análisis e interpretación de datos y la redacción final. D. Herkovits participó en la concepción y diseño del estudio, la revisión crítica del trabajo y en la aprobación final de la versión enviada para su publicación.

\section{Informaciones adicionales}

ORCID: Constanza Moretti (0000-0001-74497548); Damián Herkovits (0000-0001-6280-976X).

\section{Agradecimientos}

Nuestros agradecimientos al Instituto de Salud Colectiva de la Universidad Nacional de Lanús, al Consejo Nacional de Investigaciones Científicas y Técnicas (CONICET) y al Centro Latinoamericano de Estudios de Violencia y Salud de la Fundación Oswaldo Cruz (Fiocruz) por motivar esta investigación.

\section{Referencias}

1. Hutson E. Cyberbullying in adolescence: a concept analysis. Adv Nurs Sci 2016; 39:60-70.

2. Menesini E, Nocentini A, Paladino B, Scheithauer H, Schultze-Krumbholz A, Frisén A, et al. Definitions of cyberbullying. In: Smith P, Steffgen G, editors. Cyberbullying through the new media: findings from an international network. London/New York: Psychology Press; 2013. p. 23-36.

3. Peter IK, Petermann F. Cyberbullying: a concept analysis of defining attributes and additional influencing factors. Comput Human Behav 2018; 86:350-66.

4. Kowalski R, Giumetti G, Schroeder A, Lattanner M. Bullying in the digital age: a critical review and meta-analysis of cyberbullying research among youth. Psychol Bull 2014; 140:1073-137.

5. Espelage D, Rao M, Craven R. Theories of cyberbullying. In: Bauman S, Cross D, Walker J, editors. Principles of cyberbullying research: definitions, measures, and methodology. New York: Routledge; 2015. p. 49-64.

6. Bottino SMB, Bottino CMC, Regina CG, Correia AVL, Ribeiro WS. Cyberbullying and adolescent mental health: systematic review. Cad Saúde Pública 2015; 31:463-75.

7. John A, Glendenning AC, Marchant A, Montgomery P, Stewart A, Wood S, et al. Self-harm, suicidal behaviours, and cyberbullying in children and young people: systematic review. J Med Internet Res 2018; 20:e129.

8. Bayraktar F, Machackova H, Dedkova L, Cerna A, Ševčíková A. Cyberbullying: the discriminant factors among cyberbullies, cybervictims, and cyberbully-victims in a Czech adolescent sample. J Interpers Violence 2015; 30:3192216. 
9. Huluba Grigore AN. The dynamics of the roles of aggressor and victim in bullying and cyberbullying: a challenge for the resilient development of students. Symposion 2014; 1:223-34.

10. Allison KR, Bussey K. Cyber-bystanding in context: a review of the literature on witnesses' responses to cyberbullying. Child Youth Serv Rev 2016; 65:183-94.

11. Brochado S, Soares S, Fraga S. A scoping review on studies of cyberbullying prevalence among adolescents. Trauma Violence Abuse 2017; 18:523-31.

12. Garaigordobil M. Cyberbullying in adolescents and youth in the Basque Country: prevalence of cybervictims, cyberaggressors, and cyberobservers. J Youth Stud 2015; 18:569-82.

13. Aboujaoude E, Savage MW, Starcevic V, Salame WO. Cyberbullying: review of an old problem gone viral. J Adolesc Health 2015; 57:10-8.

14. Baldry AC, Farrington DP, Sorrentino A. "Am I at risk of cyberbullying"? A narrative review and conceptual framework for research on risk of cyberbullying and cybervictimization: the risk and needs assessment approach. Aggress Violent Behav 2015; 23:36-51.

15. Kowalski RM, Limber SP, McCord A. A developmental approach to cyberbullying: prevalence and protective factors. Aggress Violent Behav 2018; 45:20-32.

16. Pabian S, Vandebosch H. Using the theory of planned behaviour to understand cyberbullying: The importance of beliefs for developing interventions. Eur J Dev Psychol 2014; 11:46377.

17. Beyazit U, Şimşek Ş, Ayhan AB. An examination of the predictive factors of cyberbullying in adolescents. Soc Behav Pers 2017; 45:151122.

18. Paez GR. Cyberbullying among adolescents: a general strain theory perspective. J Sch Violence 2018; 17:74-85.

19. Meter DJ, Bauman S. Moral disengagement about cyberbullying and parental monitoring: Effects on traditional bullying and victimization via cyberbullying involvement. J Early Adolesc 2018; 38:303-26.

20. Twardowska-Staszek E, Zych I, Ortega-Ruiz R. Bullying and cyberbullying in Polish elementary and middle schools: validation of questionnaires and nature of the phenomena. Child Youth Serv Rev 2018; 95:217-25.

21. Betts LR, Spenser KA. Developing the cyber victimization experiences and cyberbullying behaviors scales. J Soc Psychol 2017; 178:14764.

22. Buelga S, Cava MJ, Musitu G. Validation of the adolescent victimization through mobile phone and internet scale. Rev Panam Salud Pública 2012; 32:36-42.

23. Gálvez-Nieto J, Vera-Bachman D, Cerda C, Díaz R. Escala de victimización entre adolescentes a través del teléfono móvil y de internet: estudio de validación de una versión abreviada en estudiantes chilenos. Rev Iberoam Diagn Eval Psicol 2016; 1:16-27.
24. Pérez JC, Astudillo J, Varela TJ, Lecannelier AF. Evaluación de la efectividad del programa vínculos para la prevención e intervención del bullying en Santiago de Chile. Psicol Esc Educ 2013; 17:163-72.

25. Nocentini A, Zambuto V, Menesini E. Antibullying programs and information and communication technologies (ICTs): a systematic review. Aggress Violent Behav 2015; 23:52-60.

26. Brandau M, Evanson TA. Adolescent victims emerging from cyberbullying. Qual Health Res 2018; 28:1584-94.

27. Berne S, Frisén A, Kling J. Appearance-related cyberbullying: a qualitative investigation of characteristics, content, reasons, and effects. Body Image 2014; 11:527-33.

28. Price D, Green D, Spears B, Scrimgeour M, Barnes A, Geer R, et al. A qualitative exploration of cyber-bystanders and moral engagement. J Psychol Couns Sch 2014; 24:1-17.

29. Reason L, Boyd M, Reason C. Cyberbullying in rural communities: origin and processing through the lens of older adolescents. Qual Rep 2016; 21:2331-48.

30. Noblit G, Hare R. Meta-ethnography: synthesizing qualitative studies. Newbury Park: SAGE Publications; 1988.

31. Blumer H. El interaccionismo simbólico: perspectiva y método. Barcelona: Hora; 1982.

32. Goffman E. La presentación de la persona en la vida cotidiana. Buenos Aires: Amorrortu; 1994.

33. Goffman E. Estigma: La identidad deteriorada. Buenos Aires: Amorrortu; 2006.

34. Giménez G. La discriminación desde la perspectiva del reconocimiento social. Revista de Investigación Social 2005; 1:31-45.

35. Tomasini M. Hacerse el malo: interacciones cotidianas entre estudiantes varones de primer año de escuelas secundarias de Córdoba, Argentina. Sex Salud Soc (Rio J.) 2013; (15):86112.

36. Honneth A. La lucha por el reconocimiento: por una gramática moral de los conflictos sociales. Barcelona: Crítica; 1997.

37. Ringrose J, Renold E. Normative cruelties and gender deviants: the performative effects of bully discourses for girls and boys in school. $\mathrm{Br}$ Educ Res J 2010; 36:573-96.

38. Butler J. Actos performativos y constitución del género: un ensayo sobre fenomenología y teoría feminista. Debate Feminista 1998; 18:296-314.

39. France EF, Cunningham M, Ring N, Uny I, Duncan EAS, Jepson RG, et al. Improving reporting of meta-ethnography: the eMERGe reporting guidance. BMC Med Res Methodol 2019; 19:25.

40. Bryce J, Fraser J. "It's common sense that it's wrong": young people's perceptions and experiences of cyberbullying. Cyberpsychol Behav Soc Netw 2013; 16:783-7.

41. Bryce J, Fraser J. The role of disclosure of personal information in the evaluation of risk and trust in young peoples' online interactions. Comput Human Behav 2014; 30:299-306. 
42. DeSmet A, Veldeman C, Poels K, Bastiaensens S, Van Cleemput K, Vandebosch H, et al. Determinants of self-reported bystander behavior in cyberbullying incidents amongst adolescents. Cyberpsychol Behav Soc Netw 2014; 17:20715.

43. Connolly JP. Exploring the factors influencing gifted adolescents' resistance to report experiences of cyberbullying behavior: toward an improved understanding. J Educ Gift 2018; 41:136-59.

44. Patterson LJ, Allan A, Cross D. Adolescent perceptions of bystanders' responses to cyberbullying. New Media Soc 2017; 19:366-83.

45. Stacey E. Research into cyberbullying: student perspectives on cybersafe learning environments. Informatics in Education 2009; 8:11530.

46. Varjas K, Meyers J, Kiperman S, Howard A. Technology hurts? Lesbian, gay, and bisexual youth perspectives of technology and cyberbullying. J Sch Violence 2013; 12:27-44.

47. Varjas K, Talley J, Meyers J, Parris L, Cutts H. High school students' perceptions of motivations for cyberbullying: an exploratory study. West J Emerg Med 2010; 11:269-73.

48. Dredge R, Gleeson JFM, de la Piedad Garcia $\mathrm{X}$. Risk factors associated with impact severity of cyberbullying victimization: a qualitative study of adolescent online social networking. Cyberpsychol Behav Soc Netw 2014; 17:28791.

49. Nilan P, Burgess H, Hobbs M, Threadgold S, Alexander W. Youth, social media, and cyberbullying among australian youth: "sick friends". Soc Media Soc 2015; 1:205630511560484.

50. Law DM, Shapka JD, Domene JF, Gagné MH. Are cyberbullies really bullies? An investigation of reactive and proactive online aggression. Comput Human Behav 2012; 28:664-72.

51. Betts LR, Spenser KA. "People think it's a harmless joke": young people's understanding of the impact of technology, digital vulnerability and cyberbullying in the United Kingdom. J Child Media 2017; 11:20-35.

52. Bowler L, Knobel C, Mattern E. From cyberbullying to well-being: a narrative-based participatory approach to values-oriented design for social media. J Assoc Inf Sci Technol 2015; 66:1274-93.

53. Keipi T, Oksanen A. Self-exploration, anonymity and risks in the online setting: analysis of narratives by 14-18-year olds. J Youth Stud 2014; 17:1097-113.

54. Baas N, de Jong MDT, Drossaert CHC. Children's perspectives on cyberbullying: insights based on participatory research. Cyberpsychol Behav Soc Netw 2013; 16:248-53.

55. Radovic A, Gmelin T, Stein BD, Miller E. Depressed adolescents' positive and negative use of social media. J Adolesc 2017; 55:5-15.

56. Ging D, O’Higgins Norman J. Cyberbullying, conflict management or just messing? Teenage girls' understandings and experiences of gender, friendship, and conflict on Facebook in an Irish second-level school. Fem Media Stud 2016; 16:805-21.
57. Šléglová V, Černá A. Cyberbullying in adolescent victims: perception and coping. Cyberpsychology 2011; 5:4.

58. Samoh N, Boonmongkon P, Ojanen TT, Samakkeekarom R, Jonas KJ, Guadamuz TE. 'It's an ordinary matter': perceptions of cyberbullying in Thai youth culture. J Youth Stud 2019; 22:240-55

59. Parris L, Varjas K, Meyers J. "The internet is a mask": high school students' suggestions for preventing cyberbullying. West J Emerg Med 2014; 15:587-92.

60. Parris L, Varjas K, Meyers J, Cutts H. High school students' perceptions of coping with cyberbullying. Youth Soc 2012; 44:284-306.

61. Pabian S, Erreygers S, Vandebosch H, Van Royen K, Dare J, Costello L, et al. "Arguments online, but in school we always act normal": the embeddedness of early adolescent negative peer interactions within the whole of their offline and online peer interactions. Child Youth Serv Rev 2018; 86:1-13

62. Pelfrey Jr. WV, Weber NL. Student and school staff strategies to combat cyberbullying in an urban student population. Prev Sch Fail 2015; 59:227-36

63. O’Brien N, Moules T. Not sticks and stones but tweets and texts: findings from a national cyberbullying project. Pastor Care Educ 2013; 31:53-65.

64. Ševčíková A, Šmahel D, Otavová M. The perception of cyberbullying in adolescent victims. Emot Behav Diffic 2012; 17:319-28.

65. Wright MF. Cybervictims' emotional responses, attributions, and coping strategies for cyber victimization: a qualitative approach. Safer Communities 2016; 15:160-9.

66. Maher D. Cyberbullying: an ethnographic case study of one Australian upper primary school class. Youth Stud Aust 2008; 27:50-7.

67. Vandebosch H, Van Cleemput K. Defining cyberbullying: a qualitative research into the perceptions of youngsters. Cyberpsychol Behav 2008; 11:499-503.

68. DeSmet A, Bastiaensens S, Van Cleemput K, Poels K, Vandebosch H, De Bourdeaudhuij I. Mobilizing bystanders of cyberbullying: an exploratory study into behavioural determinants of defending the victim. Stud Health Technol Inform 2012; 181:58-63.

69. Bourdieu P, Wacquant L. Una invitación a la sociología reflexiva. Buenos Aires: Siglo XXI; 2005.

70. Noel G. De los códigos a los repertorios: algunos atavismos persistentes acerca de la cultura y una propuesta de reformulación. Revista Latinoamericana de Metodología de las Ciencias Sociales 2013; 3:1-30.

71. Ministerio de Justicia y Derechos Humanos. Ciberbullying: guía práctica para adultos. http://www.jus.gob.ar/media/1039016/guiacyberbullying.pdf (accedido el 01/Abr/2020).

72. Ang RP. Adolescent cyberbullying: a review of characteristics, prevention and intervention strategies. Aggress Violent Behav 2015; 25:3542. 


\section{Abstract}

Cyberbullying is a form of online aggression between peers, the prevalence of which varies from $10 \%$ to $40 \%$ according to studies in different countries. A large share of the scientific literature on cyberbullying tends to individualize and medicalize the causes of the violence, without understanding the context in which it takes place or the meanings it acquires for those who practice it. The study aims to understand the beliefs, values, and practices that adolescents mobilize in performing the roles involved in cyberbullying. The study was conducted as a meta-ethnography, aimed at producing a synthesis of qualitative studies based on the theoretical interpretation of their basic findings. The study's corpus consisted of 33 articles selected from the BVS, PubMed, SciELO, and Scopus databases. The results include a description of expressions of cyberbullying, motivations, and adolescents' experiences as victims, perpetrators, and bystanders. With symbolic interactionism as the theoretical reference, we found that cyberbullying is a unique expression of online sociability. We contend that its practice is associated with identity-building processes, based on mechanisms of peer identification and opposition by which the participants also reproduce and compete for positions of recognition in their sociability. In this process, cyberbullying sanctions behaviors that transgress a dominant symbolic order for adolescence.

Cyberbullying; Interpersonal Relations; Violence; Adolescent; Review

\section{Resumo}

O cyberbullying é uma agressão virtual entre pares cuja prevalência varia entre 10\% e 40\%, segundo estudos desenvolvidos em diferentes países. Grande parte da bibliografia académica sobre esta agressão tende a individualizar e a medicalizar as causas da violência, deixando de contemplar o contexto no qual se dá e os sentidos que adquire para quem a exerce. Este trabalho tem como objetivo conhecer as crenças, valores e práticas que os adolescentes mobilizam ao desempenhar os papéis envolvidos no cyberbullying. Foi realizada uma meta-etnografia cujo propósito era produzir uma sintese de pesquisas qualitativas a partir da interpretação teórica dos seus achados fundamentais. $O$ corpus analisado era composto por 33 artigos selecionados nas bases de bibliografia científica BVS, PubMed, SciELO e Scopus. Os resultados descrevem as expressões do cyberbullying, os motivos que o desencadeiam e as vivências de adolescentes agressores, agredidos e observadores. Tomando como referência teórica o interacionismo simbólico, consideramos que o cyberbullying é uma expressão singular da sociabilidade virtual. Argumentamos que é uma prática relacionada com processos de construção da identidade a partir de mecanismos de identificação e oposição com pares, por meio dos quais também reproduzem e disputam posições de reconhecimento na sua sociabilidade. Neste processo, através do cyberbullying são sancionados comportamentos que infringem uma ordem simbólica dominante sobre o dever ser e estar adolescente.

Cyberbullying; Relações Interpessoais; Violência; Adolescente; Revisão
Recibido el 27/Abr/2020

Versión final presentada el 30/Oct/2020

Aprobado el 02/Dic/2020 